\title{
Recent Progress for Muon Collider
}

\section{Katsuya Yonehara ${ }^{1}$}

Fermi National Accelerator Laboratory

Batavia, IL, USA 60510

E-mail: yoneharaefnal.gov

An overview is give of results from the recent Muon Collider activities. This document begins from an introduction of the baseline design of the Muon Collider. It also mentions particularly about the progressive and essential beam elements for the Muon Collider; a proton driver based on the Project $\mathrm{X}$ and a 6-Dimensional ionization cooling channel. This document very naively touches with collider ring and detector designs.

10th International Workshop on Neutrino Factories, Super beams and Beta beams Valencia, Spain

30 June-05 July, 2008

$1 \quad$ Speaker 


\section{Introduction}

High energy and high luminosity Muon Collider (MC) can be one of the most sensitive microscopes to investigate the mechanism of hierarchy structure of elementary particles, to discover a new particle, e.g. supersymmetric particles and/or substructure of present elementally particles, and to reveal new aspects of fundamental physics. The MC provides a possible approach to a multi-TeV and luminosity of the order of $10^{34} \mathrm{~cm}^{-2} \mathrm{~s}^{-1}$ lepton collider hence a way to explore new territory beyond the reach of present and near future colliders, e.g. LHC, ILC, and CLIC.

A process to collide muons in the $\mathrm{MC}$ is as following. A positive and negative muons are generated, collected, cooled, accelerated, and finally collided each other in the MC by using following various beam elements: A high intense proton beam which is generated in a proton driver is injected into a target to produce pions in a target section. Pions are captured in a collection system and decayed into muons in a decay channel. The initial muon beam phase space after the decay channel is too large to accept in the RF accelerator system. Therefore, the muon beam is needed to be bunched and the energy-time phase space of each bunched beam is rotated to fulfill the RF bucket effectively in a phase rotation system. The initial RF frequency must be low, a few hundred $\mathrm{MHz}$, to capture the large number of muons. On the other hand, in order to accelerate muons effectively, a high frequency, a GHz region, RF acceleration field is needed. For this sake, the phase space volume must be exceptionally cooled down to the acceptance of an ILC type RF accelerator system in a cooling channel. Then, the beam is accelerated up to the required energy, and fed into a collider ring. Finally, both charged muons make collision in the collider ring. See also ref [1] for more information.

Some advanced studies have been done for some beam elements which are mentioned below subsections.

\section{Proton Driver}

A new proton superconducting (SC) linear accelerator has been proposed at Fermilab, which is called Project $X$ [2]. This machine will provide an $8 \mathrm{GeV}$ proton beam with $2 \mathrm{MW}$ beam power as a baseline design. The current $\mathrm{MC}$ requires the proton beam power around 3-4 MW. In particular, the optimum bunch length for the MC is in the range of 1-3 ns at a repetition rate in the range of $10-60 \mathrm{~Hz}$. The Project X need to be upgraded to fulfill those parameters.

The intensity capability of Project X must be enhanced to achieve the 4 MW beam power. Aiming for power levels even beyond the 4-MW level would seem prudent. We need to study performing evaluations of instabilities in analytic and simulation studies. We also need to carry out tracking studies including realistic errors.

Because the proposed MC repetition rate is high, it is worthwhile to consider the technical implications of implementing the Project $\mathrm{X}$ linac as a $\mathrm{CW}$ device. We need the same study as above, instability analysis and tracking studies including realistic errors. More ambitiously, reuse of the CW linac to accelerate muons is considered [3]. 


\section{Six-Dimensional Phase Space Cooling System}

Muons which inject in a matter isotropically lose their kinetic energies by an ionization energy loss process. The kinetic energy is recovered by the RF accelerator field. As a result, the phase space volume shrinks in the transverse plane. It is called ionization cooling. Gaseous and liquid hydrogen (GH2 and LH2), lithium hydride ( $\mathrm{LiH}$ ) and some low-Z metals ( $\mathrm{Li}, \mathrm{Be}$ ) can be used for the ionization cooling absorber. To achieve 6-Dimensional (6D) cooling it requires swapping the longitudinal and transverse degrees of freedom. It is called emittance exchange. It can be made by tuning the path length in the cooling absorber by using a dispersive beam element.

One of the most attractive 6D cooling channels is a helical cooling channel (HCC). The HCC is consisted of a helical dipole, helical quadrupole magnetic components and a solenoid component is superimposed. The helical dipole and solenoid components provide a continuous dispersion. The HCC can provide a continuous emittance exchange as well as the continuous ionization cooling with incorporating a sequential high pressure gaseous hydrogen filled RF cavity into the HCC. Consequently, the muon beam phase space is cooled effectively [4]. To incorporate the RF structure into the HCC is one of the challenging issues.

Another progressive design of the 6D cooling channel is the RFOFO ring channel. It uses the alternate solenoid field to generate a low beta function. Thus, the lower phase space than the HCC with the same field strength can be obtained. There is a big improvement in the current design; a ring shape turns out to be the helical structure to avoid the beam injection and extraction problem. It is called Guggenheim channel [5]. The evacuated type RF system cannot be operated under a high magnetic field. One of big issues in this channel is how to isolate the field on the RF system.

\section{Collider Ring and Detector}

The momentum spread in the collider ring is expected to be $\delta \mathrm{p} / \mathrm{p}=0.1 \sim 1 \%$ at a few $\mathrm{TeV}$, that is similar as the designed values in ILC and CLIC. Therefore, the basic designs of MC ring and detector can be referred to the ILC and CLIC. This activity has just begun [6].

\section{References}

[1] S. Osaki et al., Feasibility Study-II of a Muon Based Neutrino Source, BNL-52623 (2001), also available at http://www.cap.bnl.gov/mumu/studyii/

[2] See http://projectx.fnal.gov

[3] R.P. Johnson et al., A FERMILAB CW LINAC WITH HIGH POTENTIAL BEAM POWER, XXIV Linear Accelerator Conference (LINAC08) September 29-October 3, 2008, to be published

[4] Y. Derbenev and R.P. Johnson, Six-dimensional muon beam cooling using a homogeneous absorber: Concepts, beam dynamics, cooling decrements, and equilibrium emittances in a helical dipole channel, PRSTAB 8, 041002 (2005)

[5] See http://www.muonsinc.com/mcwfeb06/presentations/AKlier_02072006_Guggenheim.pdf

[6] See http://www.muonsinc.com/lemc2008 to find presentations related with the MC ring and detector designs 